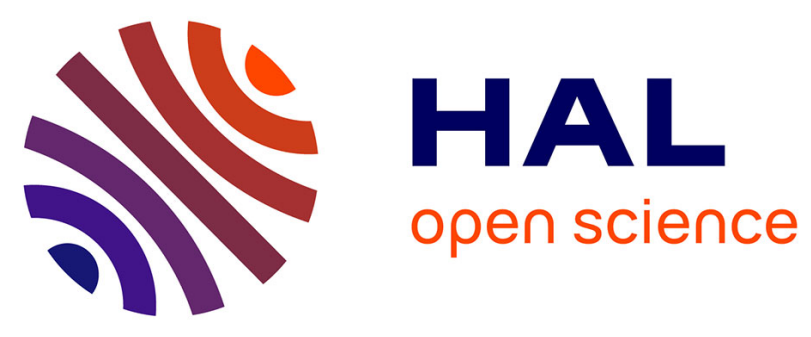

\title{
Rethinking Wearables in the Realm of Architecture
}

\author{
Himanshu Verma, Hamed S. Alavi, Denis Lalanne
}

\section{To cite this version:}

Himanshu Verma, Hamed S. Alavi, Denis Lalanne. Rethinking Wearables in the Realm of Architecture. 16th IFIP Conference on Human-Computer Interaction (INTERACT), Sep 2017, Bombay, India. pp.16-23, 10.1007/978-3-319-92081-8_2 . hal-01821409

\section{HAL Id: hal-01821409 \\ https://inria.hal.science/hal-01821409}

Submitted on 22 Jun 2018

HAL is a multi-disciplinary open access archive for the deposit and dissemination of scientific research documents, whether they are published or not. The documents may come from teaching and research institutions in France or abroad, or from public or private research centers.
L'archive ouverte pluridisciplinaire HAL, est destinée au dépôt et à la diffusion de documents scientifiques de niveau recherche, publiés ou non, émanant des établissements d'enseignement et de recherche français ou étrangers, des laboratoires publics ou privés.

\section{(c)(1)}

Distributed under a Creative Commons Attribution| 4.0 International License 


\title{
Rethinking Wearables in the Realm of Architecture
}

\author{
Himanshu Verma, Hamed S. Alavi, and Denis Lalanne \\ Human-IST Research Institute \\ University of Fribourg \\ Boulevard de Pérolles 90, 1700 Fribourg, Switzerland \\ \{himanshu.verma, hamed.alavi, denis.lalanne\}@unifr.ch
}

\begin{abstract}
The architectural built environments, which so ubiquitously, act as shelters and shape our daily personal and social experiences, can soon be envisioned as being interacted with and mediated through wearables. This conjecture is becoming salient with the increased interactivity of our built environments, and a sustained drive to render them energy efficient. This entails for the upscaled re-design, appropriation, and assessment of functions that are typically ascribed to wearable technologies, as well as the grounding of users' sociotechnical interactions and experiences within the built environments.

In this position paper, we discuss this inevitable shift in the role of wearables and the expansion of its functional spectrum to include the built environments and the constituent social constructs, thus facilitating a comprehensive experience of inhabitants' well-being.
\end{abstract}

Keywords: well-being, human-building interaction, sustainable HCI

\section{$1 \quad$ Introduction}

Le Corbusier, in his 1923 book Vers une architecture, has referred to a building as a machine to inhabit. This perspective is growing ever more relevant with the continued accelerated measures to increase the efficiency of built environments in terms of energy consumption and performance. Consequently, existing built environments are increasingly retrofitted with interactive elements (for example, NEST thermostat ${ }^{1}$ ) to optimize energy usage by $a$ ) automating specific functions, b) providing awareness about (the consequences of) inhabitants' actions, and c) providing a platform (in a long term) to change one's behavior towards energy-efficient living. Additionally, in newly constructed buildings, more specifically the one certified by low-energyconsumption standards (for example, Minergie in Switzerland), automated heating and ventilation systems have mandated the removal of operational windows. While these developments have been reported to be advantageous in conserving energy, the lack of control over the environment (as a consequence of automation) has raised concerns about the inhabitants' perceived comfort [1], [2]. Furthermore, the furnish-

1 https://nest.com/thermostat/meet-nest-thermostat/ 
ing of varied interactive and awareness devices calls for the design, appropriation, and assessment of new interaction paradigms and socio-technical practices. These evolving concerns and opportunities entail the monitoring of environmental parameters, knowledge about existing social constructs, and acute context awareness followed by recommendations for contextualized actions on the part of both the built environment and the inhabitants.

The functions of continued observance, diagnosis, and awareness of individuals' physical or physiological state are already ascribed to numerous wearable devices (for example fitness and activity trackers, medical implants, etc.). We believe that with the evolution of our built environments, these aforementioned functions are being expanded to include, beyond just the physiological state, the (spatio-temporal) knowledge about our physical environment and social contexts. Consequently, we can envision supplementing the role of wearables as a facilitator for our (two-way) interactions with the built environments and other inhabitants, in a way that our living/working experiences are grounded within an ecosystem of socio-technical systems comprising of sensors, actuators, ambient information, and data analyses. Furthermore, a (multi-modal) data-centric approach may manifest in the "quantified home (or office)" as an extension of the lifelogging movement.

In the following sections, we will illustrate this notion with the (developing) perspective of Human-Building Interaction (HBI) [3] [4], as well as our own participatory experiences within an interdisciplinary living lab project comprising of architects, building performance researchers, designers, and us (HCI researchers).

\section{Human-Building Interaction (HBI)}

Human-Building Interaction (HBI) is an emerging notion at the intersection of architecture, interaction design, and UbiComp that aims "to provide interactive opportunities for the occupants to shape the physical, spatial, and social impacts of their built environments" [3]. Interaction design and UbiComp have on numerous occasions drawn inspirations from the domains of architecture and urban design. However, the concrete possibilities for these domains to closely work together have been rare in the past [4]. HBI is (consequently) an attempt to bring together researchers from these contributing domains to share knowledge and work in close cooperation, in order to design for the sustainable living experiences while addressing the evolving living and working styles and habits of inhabitants. Smart Living Lab, as discussed in the next section, is a unique project which is the manifestation of principles at the core of HBI.

\subsection{Smart Living Lab}

Smart Living $\mathrm{Lab}^{2}$ is an inter-disciplinary lab engaged in the envisioning of the built environments of the future by examining the research questions that concern sustainable living and working experiences, which are grounded in the evolving socio-cultural

2 https://smartlivinglab.ch 
practices. A prototype building was constructed to study these aspects in central Switzerland, in the bilingual city of Fribourg. This building currently serves as a workspace for around 100 researchers from three academic institutions - Swiss Federal Institute of Technology (EPFL), University of Fribourg, and School of Engineering and Architecture in Fribourg (HEIA). The researchers come from different domains of Architecture, Building Physics and Performance, Design, Law, and HCI. Amongst multiple projects that are currently being pursued, inhabitants' well-being and the perception of comfort within the changing landscape of modern architecture, as well as the role of human factors in building design are the research topics which we are currently investigating.

Our contributions within the Smart Living Lab, so far have focused on the ecologically valid and multivariate building-data visualization, exploration and analysis, which may reveal varied aspects of occupants' behavior in different scenarios. This data-centered approach has implications for the comprehension of occupants' wellbeing, and simultaneously augment it through well-grounded socio-technical interactions and experiences. Here, we believe that the wearable technologies have a crucial part to play.

\section{Beyond Quantified Self - Quantified Buildings}

The potential expansion of wearables' functional spectrum to include our built environment may augment our self-awareness about our well-being by including aspects of comfort and its perception, as well as the contextualized negotiation of environmental state (temperature, air quality, etc.) with fellow inhabitants while maintaining a sustainable living practice. This entails design, appropriation, and evaluation of new interaction mechanisms with the built environment, either directly or indirectly through wearables by examining the dynamic socio-cultural practices through an amalgamation of ethnography, interaction design, and (sensor-) data analytics. In this section, we illustrate the varied dimensions and scenarios that may constitute this shift in our fine-grained awareness beyond ourselves to our environment. These dimensions correspond to the multiple sources of available physical and physiological data from sensors, which can facilitate the acquisition of knowledge about the context (number of inhabitants, ongoing activity, physiological history, etc.), and offer a quantitative platform to negotiate environmental state between a human and a building (for example POEM [1]), or amongst inhabitants themselves. In the following sections, we will especially focus on dimensions pertaining to the quality of indoor environment and mobility of its individuals, as these are the dimensions which can afford an extension to the conventional wearables.

\subsection{Environmental Characteristics}

Environmental characteristics concerning well-being can be grouped into four categories of thermal, respiratory, visual, and acoustic well-being [5], [6]. While significant amount of research has been conducted in different domains on the thermal and res- 
piratory aspects, relatively less work accounts for the visual and acoustic aspects. This can be attributed to the immediately perceived effects of thermal environment (temperature, humidity, and air-flow) [7], and the adverse effects of inferior respiratory environment owing to the increased concentration of gases such as Carbon Dioxide, pollutants and particulate matter [8], [9]. The relevance of the respiratory environment is further heightened due to the adverse effects of poor air quality on human health, the lack of awareness about the air quality within buildings [10], [11], and especially crucial in metropolitan cities which experience increased levels of pollution.

Sensors recording different attributes of these dimensions can be distributed within the built environment, which in tandem with ambient and distributed awareness tools may prove to be informative to the inhabitants. Subsequently, these awareness (and visualization) tools can offer informed recommendations to the inhabitants about the set of possible actions (for example opening windows to allow for cross-ventilation). In addition, they can also provide an interactive platform for inhabitants to negotiate their comfort parameters (for example, in case of conflicts resulting from varying thermal perceptions). ComfortBox by Alavi et al. [12] is an example of such a tool that affords for awareness about these four dimensions, a possibility to inform the building (through interactions) about levels of acceptable comfort, and communicate one's perceived comfort to other inhabitants.

The perceived loss of control over different architectural elements such as windows and shades, with the increased automation of buildings and its environment, has also been observed by Brambilla et al. [2] to negatively influence the perceived wellbeing of inhabitants. This further exacerbates the need for tools and mechanisms that can mitigate this negative perception by providing awareness, empowering inhabitants to express their opinions about their well-being, and eventually enabling them to negotiate the desired environment. Furthermore, we envision that the living experience can be enriched by combining physiological information from conventional wearables (for example, body temperature, skin conductance), as it can facilitate the acquisition of precise knowledge about an inhabitant's health status, and enable the development of personalized and contextualized well-being models.

\subsection{Presence and Proximity}

Presence (or absence) of inhabitants within home, office, or a specific room, or proximity to certain artifacts and architectural elements within the building are vital resources to establish a precise context awareness for the built environment. Smart home technologies (for example, NEST thermostat, smart lighting) leverage this information to regulate the environmental state by controlling the HVAC (Heating, Ventilation, and Air Conditioning), or lighting systems, and consequently optimize the energy consumption. These systems often use the geo-location information of an inhabitant's smart phone to accomplish their goals.

Furthermore, at a finer level of granularity, the presence information can be used to precisely model the context such as the number of inhabitants and thus the likely activity the inhabitants are engaged in, or in specific cases to detect unexpected behavior for security reasons. Indoor localization techniques can be used to access this in- 
formation. Besides allowing for finer control over the environmental attributes, the presence information can also be used to assess various building functions in the postoccupancy phase of a building. The study conducted by Verma et al. [13] employed presence information (specifically indoor mobility) to assess if the rooms within an office building were used to their full capacity, and how inhabitants with different professional profiles contributed to the utilization of office space. Such studies, in the short term, allow for the sustainable use of buildings and provide implications for design and appropriation. In the medium term, they can act as valuable knowledge resources for the next phase of building life-cycle, and in the long term they contribute to the repertoire of knowledge about human factors in built environment. Therefore, the presence and proximity information can be leveraged with the wearables to attain a comprehensive understanding of inhabitants' behavior, and this can be utilized extensively to expand wearables' functionality beyond health monitoring to design contextualized interactive services and tools.

\subsection{Social Attributes}

Environmental attributes and inhabitants' localized information can be easily accessed through a combination of distributed sensors, and can be simultaneously leveraged to interact with our built environments. In addition, social cues and signals (such as speech times, turn-taking, proximity to other inhabitants, etc.) which constitute an integral aspect of human communication, can further enrich the contextualized knowledge of the built environment. These social aspects which are investigated and designed for by researchers in the CSCW (Computer Supported Collaborative Work) community can be utilized, either directly or indirectly, by wearables to enable occupants to exercise fine control over buildings. Furthermore, the abundant knowledge within the CSCW community, which was acquired through the analysis of social interactions (verbal and non-verbal) may foster the design of collective awareness tools (about environmental factors - both indoor and outdoor). Such tools, in the short term may allow the occupants to negotiate their comfort levels, and in the long term can motivate occupants to regulate their behavior for a sustainable living experience.

\section{Sketching the Research Landscape}

Following the illustration of the broad constellation of variables (physical, physiological, social) that can - individually or in combination - influence our immersive experiences with or within our built environments, the next logical step would be to answer questions concerning inhabitants' needs, expectations, and mental models about the nature of interaction, interactivity, and immersiveness within our environments. In addition, discussions need to be organized around the role of wearables in mediating our interactions with the built environments, how this mediation will scale with the changing landscape of socio-cultural contexts, and where (within the broad spectrum of dynamic user experiences) will the wearables contribute significantly. This entails the establishment of an interdisciplinary knowledge about perception of environmen- 
tal attributes, user experience, and inhabitant behavior through research efforts at the intersection of computer science, architecture, psychology, and sociology. In this section, we attempt to sketch the future research landscape by asking questions concerning the following themes, answers to which will concretely consolidate the role of wearable technologies in human-building interaction (HBI).

\subsection{Agency or Control}

The evolution of architecture through the incorporation of interactive and robotic elements (such as window blinds), is bringing to the forefront, the issues related to the inhabitants' agency or the sense of control over the built environment. This entails raising questions that are fundamental to the ascribed behaviors and afforded functions of wearables embodied within our interactive experiences with the built environments. For example, how do we bridge the gap between the individuality of wearables and the commonality of architecture? How can the coupling of wearables and adaptive/robotic environments provide for a seemingly integrative experience to the users? Or what features can be extracted from wearable sensing that could shed light on the points of inefficiency in the interaction between built environments and their occupants? Furthermore, how can our perception of environmental factors and our subjective experiences be leveraged and integrated by the wearables to enhance the overall user experience? In social scenarios, how can the agency be shared between co-habitants, or delegated to a specific inhabitant?

One of the HBI challenges is to reconcile inhabitants' agency with the efficiency of building automation systems. So, how can wearables help address this challenge? Also, how can the wearables be designed as persuasive platforms to encourage inhabitants to pursue sustainable and environmental friendly behaviors, for example, through the awareness about the consequences of their actions?

\subsection{Health and Awareness}

Consolidating the monitoring of inhabitants' health and well-being and their awareness can easily be regarded as the extension of wearables' capabilities to include the environment. However, this extended functionality has to be well-grounded within the context (location, activity, weather, etc.), physiological state, subjective preferences, and the social context of inhabitants. In addition, how can the wearables in-tandem with other environmental sensors contribute in the understanding of personalized comfort models/zones of the inhabitants? How do these personalized models differ in varying socio-cultural contexts? How can the wearables make inhabitants' more aware of their environmental attributes, especially about the health critical attributes such as air quality? When and how should the wearables intervene as the environmental attributes change (possibly from comfortable to relative discomfort), and what recommendations about the possible actions should they offer?

Next, framing the issue of health awareness within the notion of biopower and biopolitics [14] [15], which examines the "truth discourses" about human body, health, and life, and the "authorities which are competent enough to convey this truth" such 
as the wearable technologies and the role of data in assessing our health. Biopolitics, further examines the authority around the discourses related to life and health by questioning the role of authority itself and who can be considered as an authority. For example, does technology has an authority over our experiences related to health and well-being within the built environments, or the inhabitants themselves? These questions have to be grounded and studied within the architectural context while designing wearables to mediate/augment our experiences with our built environments.

\subsection{Privacy and Data Protection}

Owing to the a) massive amount of data that is collected through the wearables and the ambient sensors within the built environment, $b$ ) its storage and analysis on (cloud) servers, $c$ ) extraction of health-related features, and $d$ ) the potential inference of the state of the inhabitants' health and its relationship to the medical history and the personalized models of comfort as well as the context-sensitive information, render privacy and data protection a critical aspect of the design and application of wearables within architectural environments. These issues entail for the examination of privacy as a fundamental right since the beginning of the design process and not as a byproduct, for example privacy by design [16] [17]. Who owns the data (inhabitants, building, or both), or has the authority over the actions resulting from data analysis and inference? In what circumstances or contexts, can the data be shared with external agents, and to what extent? How will the privacy and data protection policies evolve in varying social scenarios (for example, while hosting guests)? Going beyond the user consent, strategies and policies should be developed to evaluate the inherent risks and prevent the misuse of personal data without compromising the quality of service and the user experience.

\section{Conclusion}

In this position paper, we have argued that with the increased interactivity of our built environments and an enhanced need for sustainable living, the well-being (or comfort) of inhabitants is being rendered crucial. Here, we believe, that the wearable technologies can play a vital role in diluting the boundaries of self to (also) include our built environments. This extension in the functional spectrum of wearables is happening with the increased diffusion of different sensors (recording the thermal, respiratory, acoustic, and visual aspects of built environments), and the increasing interactivity of our living and working spaces. Furthermore, the physiological data that is being collected and analyzed by wearable devices can be combined and communicated with our built environments to $a$ ) maintain a precise awareness of the context (inhabitants' health status and the ongoing activity), $b$ ) increase the inhabitants' awareness about the environmental factors and their influence on health, $c$ ) enable a fine grained control over our built environments while optimizing the energy consumption, $d$ ) provide inhabitants with a platform to negotiate their comfort with others, and thus $e$ ) foster an enhanced living and working experience. Finally, we have 
drawn out the emerging research landscape as a consequence of the diffusion of wearable devices within the interactive experiences with our built environments, by raising relevant questions concerning agency, well-being, awareness, and privacy.

\section{Bibliography}

[1] M. Milenkovic, U. Hanebutte, Y. Huang, D. Prendergast and H. Pham, "Improving User Comfort and Office Energy Efficiency with POEM (Personal Office Energy Monitor)," in CHI'13 Extended Abstracts on Human Factors in Computing Systems, Paris, France, 2013.

[2] A. Brambilla, H. Alavi, H. Verma, D. Lalanne, T. Jusselme and M. Andersen, "'Our inherent desire for control": A case study of automation's impact on the perception of comfort," in CISBAT'17 - Future Buildings and Districts - Energy efficiency from Nano to Urban Scale, Lausanne, Switzerland, 2017.

[3] H. S. Alavi, D. Lalanne, J. Nembrini, E. Churchill, D. Kirk and W. Moncur, "Future of human-building interaction," in Proceedings of the $2016 \mathrm{CHI}$ Conference Extended Abstracts on Human Factors in Computing Systems, San Jose, 2016.

[4] H. S. Alavi, E. Churchill, D. Kirk, J. Nembrini and D. Lalanne, "Deconstructing human-building interaction," Interactions, vol. 23, no. 6, pp. 60-62, 2016.

[5] P. M. Bluyssen, M. Aries and P. van Dommelen, "Comfort of workers in office buildings: The European HOPE project," Building and Environment, vol. 46, no. 1, pp. 280-288, 2011.

[6] D. Hawkes, The environmental imagination: technics and poetics of the architectural environment, Taylor \& Francis, 2008.

[7] P. O. Fanger, Thermal comfort. Analysis and applications in environmental engineering., Copenhagen: Copenhagen: Danish Technical Press., 1970.

[8] C.-G. Bornehag, J. Sundell, C. J. Weschler, T. Sigsgaard, B. Lundgren, M. Hasselgren and L. Hägerged-Engman, "The association between asthma and allergic symptoms in children and phthalates in house dust: a nested case-control study," Environmental health perspectives, vol. 112, no. 14, p. 1393, 2004.

[9] W. J. Fisk, Q. Lei-Gomez and M. J. Mendell, "Meta-analyses of the associations of respiratory health effects with dampness and mold in homes," Indoor Air, vol. 17, no. 4, pp. 248-296, 2007.

[10] S. Kim and E. Paulos, "inAir: measuring and visualizing indoor air quality," in Proceedings of the 11th international conference on Ubiquitous computing, Orlando, Florida, 2009. 
[11] M. Frešer, A. Gradišek, B. Cvetković and M. Luštrek, "An intelligent system to improve THC parameters at the workplace," in Proceedings of the 2016 ACM International Joint Conference on Pervasive and Ubiquitous Computing: Adjunct, Heidelberg, Germany.

[12] H. S. Alavi, H. Verma, M. Papinutto and D. Lalanne, "'Comfort": A coordinate of User Experience in Interactive Built Environments," in Interact'17, Mumbai, India, 2017.

[13] H. Verma, H. S. Alavi and D. Lalanne, "Studying Space Use: Bringing HCI Tools to Architectural Projects," in Proceedings of the 2017 CHI Conference on Human Factors in Computing Systems, Denver, Colorado, USA, 2017.

[14] M. Foucault, "The Birth of Biopolitics," in Ethics, Subjectivity, and Truth, 1997.

[15] P. Rabinow and R. Nikolas, "Biopower today," in BioSocieties, 2006.

[16] M. Langheinrich, "Privacy by design - principles of privacy-aware ubiquitous systems," in Ubicomp 2001: Ubiquitous Computing, 2001.

[17] A. Cavoukian, "Privacy by design," in Take the challenge. Information and privacy commissioner of Ontario, Canada. 